\title{
ENSINAR A TODOS E A CADA UM EM ESCOLAS INCLUSIVAS: a abordagem do ensino diferenciado
}

Keisyani da Silva Santos Enicéia Gonçalves Mendes

Resumo

$\mathrm{Na}$ medida em que os sistemas escolares foram se tornando mais acessíveis à população o ensino foi sendo rapidamente massificado e as políticas de inclusão escolar surgidas, a partir dos anos de 1990, ressaltaram a incapacidade de as escolas se adequarem aos desafios de sua época: oferecer qualidade de ensino a todos os alunos. O presente ensaio tem como objetivo apresentar o referencial teórico da Abordagem do Ensino Diferenciado (AED) que visa responder às diferentes necessidades educacionais dos alunos. $\mathrm{O}$ texto apresenta a conceituação, os princípios, os fatores e elementos passiveis de diferenciação do ensino (conteúdo, processo, produto e gestão da aula). Destaca-se que a AED se propõe a contemplar a diversidade, melhorando o ensino para todos os alunos, indistintamente, e que, por isso, pode ser referencial potente para programas de desenvolvimento profissional de professores.

Palavras-chave: inclusão escolar; práticas pedagógicas; ensino diferenciado.

\section{TEACHING EVERYONE AND EACH ONE IN INCLUSIVE}

SCHOOLS:

the differentiated instruction approach

\begin{abstract}
As school systems became more accessible to the population, education was rapidly widespread, and school inclusion policies that emerged from the 1990s onwards highlighted the inability of schools to adapt to the challenges of their time: offering quality teaching to all students. This essay aims to present the theoretical framework of the Differentiated Instruction Approach (DIA) that aims to respond to the different educational needs of students. The text present the conceptualization, the principles, the factors and elements liable to differentiate the teaching (content, process, product and classroom management). It is noteworthy that the DIA proposes to contemplate diversity, improving teaching for all students, without distinction, and that for this reason it can be a powerful reference for professional development programs for teachers.
\end{abstract}

Keywords: school inclusion; pedagogical practices; differentiated teaching.

\section{ENSEÑAR A TODOS Y CADA UNO EN ESCUELAS INCLUSIVAS:} el enfoque de enseñanza diferenciado

Resumen

A medida que los sistemas escolares se volvieron más accesibles para la población, la educación se generalizó rápidamente y las políticas de inclusión escolar que surgieron a partir de la década de 1990 pusieron de relieve la incapacidad de las escuelas para adaptarse a los desafíos de su época: ofrecer una enseñanza de calidad a todos los estudiantes. Este ensayo tiene como objetivo presentar el marco teórico del Enfoque de Enseñanza Diferenciado (EED) que tiene como objetivo dar respuesta a las diferentes necesidades educativas de los estudiantes. El texto se organizó con el fin de presentar la conceptualización, los principios, los factores y elementos susceptibles de diferenciar la enseñanza (contenido, proceso, producto y gestión 
del aula). Es de destacar que la EED se propone contemplar la diversidad, mejorando la docencia para todos los estudiantes, sin distinción, y que por ello puede ser un referente poderoso para los programas de desarrollo profesional para docentes.

Palabras clave: inclusión escolar; prácticas pedagógicas; enseñanza diferenciada.

\section{INTRODUÇÃO}

Cada estudante é um indivíduo único, nasce, cresce e se desenvolve de maneira própria, possuindo pontos fortes, fraquezas e diferentes tradições culturais. Essa diversidade se reflete, também, nas diferenças de grupos em relação ao gênero, etnia, renda familiar e condições de moradia, por exemplo, mas decorre também de características individuais, tal como o nível e tipo de inteligência, a personalidade, habilidades físicas, etc. No caso dos estudantes do público-alvo da educação especial (PAEE), à diversidade típica somam-se outras que podem resultar em barreiras cognitivas, acadêmicas, sensoriais, físicas, nas habilidades sociais ou comportamentais, atrasos gerais no desenvolvimento etc.

Durante muitos anos a educação especial se centrou em intervir nos impedimentos dos alunos e nas habilidades consideradas deficitárias, até que eles passaram a ingressar em escolas comuns e as práticas pedagógicas tradicionais da educação especial e da educação geral entraram em conflito, trazendo o desafio aos professores de dar acesso aos conhecimentos previstos no currículo padrão a todos os estudantes. Entretanto, na prática, isso resultou em poucas mudanças e, embora haja alunos que fiquem aquém enquanto outros excedem as expectativas de seus professores, a maioria tem desempenho mediano e, de modo geral, o ensino tem se voltado para estes últimos, tomados como padrão, sem contemplar a diversidade dos alunos. Contudo, vários autores têm enfatizado a necessidade de as escolas contemplarem essa diversidade a fim de atingir a tarefa de promover ao máximo o potencial de aprendizado de todos os alunos em turmas heterogêneas (PERRENOUD,1999; HEACOX, 2006; ROLDÃO, 2003).

Nessa direção, encontram-se na literatura várias propostas que têm em comum a tese de que as práticas pedagógicas dos professores precisam responder às necessidades diversificadas dos alunos. Termos como pedagogia diferenciada, diferenciação curricular, ensino diferenciado e diferenciação do ensino, apesar de partilharem a mesma ideia, partem de abordagens teóricas/filosóficas e propostas de transposição para a prática que nem sempre convergem entre si. O presente texto tem como objetivo apresentar, a partir de uma pesquisa bibliográfica pautada na revisão da literatura, a abordagem denominada como Differentiated Instruction, aqui traduzida como Abordagem do Ensino Diferenciado (AED), que tem como principal referência teórica as produções da pesquisadora dos EUA, Carol Ann Tomlinson, da Universidade da Virgínia. Destacase que esta não é uma proposta exclusivamente direcionada para o PAEE, mas sim, visa contribuir para que os professores compreendam e respondam proativamente às diferenças de todos os seus alunos. (TOMLINSON, 1999, 2001, 2004, 2016, 2017; TOMLINSON et al, 2003; TOMLINSON, MCTIGHE, 2006; TOMLINSON, BRIMIJOIN, NARVAEZ, 2008; SOUSA, TOMLINSON, 2010).

A importância dessa abordagem se deve à capacidade da referida autora de traduzir o conceito de diferenciação em termos práticos e, assim, oferecer elementos valiosos para os professores sobre como conduzir o ensino para contemplar a diversidade de seus alunos. 


\section{OS PRINCÍPIOS DO ENSINO DIFERENCIADO (ED)}

Para Tomlinson (1999, 2008), a AED está fundamentada em cinco princípios básicos que devem ser articulados e compreendidos como complementares: currículo de qualidade; avaliação contínua; respeito às habilidades e interesses dos alunos; agrupamentos flexíveis; e comunidade de aprendizagem.

A qualidade do currículo se relaciona com a forma como os professores qualificam os objetivos da aprendizagem. A avaliação contínua diz respeito à forma como os professores pesquisam e coletam informações sobre os seus alunos e as usam para direcionar os objetivos, as aprendizagens e habilidades para responder apropriadamente às necessidades deles. O terceiro princípio implica em lançar mão de tarefas que respeitem as habilidades e interesses dos alunos, que se relacionem com a ampla escolha de atividades, com o nível de preparo, interesses e tendências dos alunos. Agrupamentos flexíveis dizem respeito à forma como o professor utiliza, na aula, uma variedade de configurações para garantir que os alunos tenham a mesma oportunidade de aprender e possam trabalhar com todos os colegas. Finalmente, comunidade de aprendizagem implica em construir um ambiente que faça com que os alunos se sintam felizes, confortáveis, aceitos e apoiados em seus processos de aprendizagem.

Tomlinson $(2001,2008)$ destaca que a AED é proativa, mais qualitativa do que quantitativa, oportuniza múltiplas formas de acesso (ao conteúdo, ao processo e ao produto), centra-se no aluno, embasa-se na avaliação, adota várias formas de abordar conteúdo/processo/produto, mescla instrução para a turma com individualização, é organizada e dinâmica. Em termos práticos, esta abordagem é compreendida como um processo cíclico e contínuo de conhecer os alunos e responder pedagogicamente com diferenciação (TOMLINSON, 2008; HEACOX, 2006) e esses dois fatores darão materialidade às premissas de valorização e respeito à heterogeneidade em sala de aula.

\section{ENSINO DIFERENCIADO: COMO CONHECER OS ALUNOS}

Diversos são os fatores que influenciam a aprendizagem dos alunos, como por exemplo: as experiências escolares anteriores, o contexto familiar, social, cultural e econômico, os suportes e recursos que dispõem, as condições emocionais, suas habilidades e dificuldades. Muitos destes fatores estão além do controle do professor, mas se ele obtiver conhecimento sobre quem é o seu aluno e como este aprende melhor, poderá organizar o ensino de modo que eles aprendam mais e com maior qualidade (SOUZA, TOMLINSON, 2011). Assim, na AED o processo de conhecer os alunos deve ser intencionalmente planejado como parte integrante do plano de ensino para a turma e, por isso, o professor planeja e realiza avaliações sistemáticas e periódicas, com o objetivo de conhecer toda turma e a cada integrante. Por isso, a avaliação contínua e formativa é primordial para a qualidade e a efetividade da diferenciação

Assim, conhecer os alunos com base em avaliações é um pressuposto primordial, pois, neste processo avaliativo estão contidos os três fatores que orientam a diferenciação do ensino: nível de preparação, interesses e perfil de aprendizagem.

\section{Avaliação do Nível de Preparação}

Souza e Tomlinson (2011, p. 85) definem o nível de preparação como “[...] proximidade atual de um indivíduo ou a proficiência em um conjunto específico de conhecimento, 
entendimento e habilidades designadas como essenciais para um segmento específico de estudo". Assim, nível de preparação não é sinônimo de pré-requisitos, mas se aproxima do conceito de Zona de Desenvolvimento Atual de Vigotski, que consiste naquilo que o sujeito é capaz de realizar sozinho, sem a mediação do outro, a qual "[...] revela não apenas as funções amadurecidas, mas também 'apalpa' as funções em amadurecimento" (PRESTES, 2010, p. 170) e do conceito de Zona de Desenvolvimento Iminente, a qual consiste naquilo em que o sujeito necessita da mediação do outro para realizar. Enfim, este conceito ressalta a estreita ligação "[...] existente entre desenvolvimento e instrução e a ação colaborativa do outro” (PRESTES, 2010, p. 168).

No processo de avaliação do nível de preparação, o professor deve investigar habilidades em diversos âmbitos, como por exemplo: o conhecimento prévio, a complexidade das ideias, níveis de representação e aplicação, autonomia, o tipo de estrutura que melhor responde aos objetivos e ao ritmo de estudo e raciocínio (TOMLINSON, 2008).

Assim, conhecer os níveis de preparação dos alunos permite que o professor planeje o ensino, adequadamente, para que esteja de acordo com suas habilidades atuais, possibilitando seu avanço. Cabe destacar que os alunos podem apresentar níveis de preparação diferentes no conteúdo do currículo e isso requer ajuste entre padrão de entrega das tarefas, conteúdos, tipos de comunicação e nível de desempenho requerido para o aluno.

\section{Avaliação dos Interesses}

De acordo com Souza e Tomlinson (2011, p. 112), o fator interesse "[...] refere-se a um sentimento ou emoção que faz com que o sujeito se concentre ou entenda algo". Temas, eventos, atividades e objetos que despertem o interesse de um estudante são capazes de atrair e prender a atenção, mantendo-o concentrado por mais tempo, além de instigar a curiosidade. O interesse em aprender é compreendido como um conjunto de comportamentos manifestos quando uma pessoa está envolvida em uma atividade, sendo eles: maior atenção, concentração, sentimentos agradáveis ao esforço despendido, maior disposição, persistência, energia e intensidade (SOUZA; TOMLINSON, 2011).

A avaliação dos interesses deve ser abrangente, abarcando diversas áreas, como por exemplo: interesses pessoais, de lazer e entretenimento, profissionais, acadêmicos, em artes plásticas, poesia, escrita, tecnologia, ciências sociais, organizações e grupos, ciências naturais, música, dramatizações e jogos, esportes, artes manuais, oratória, atividades sociais, entre outras.

\section{Avaliação do perfil de aprendizagem}

Todos são capazes de aprender de diversos modos e percorrendo diferentes caminhos, entretanto, alguns modos e caminhos podem favorecer a aprendizagem de um aluno e dificultar a aprendizagem de outro. O perfil de aprendizagem corresponde à forma como os estudantes tendem a aprender mais rápido e com melhor qualidade e refere-se às preferências pessoais e ambientais do sujeito. São compreendidas como adquiridas e adaptáveis, logo, não são inatas e imutáveis (SOUZA, TOMLINSON, 2011; TOMLINSON, 2001, 2008). Para compor este perfil a proposta da AED se baseia em quatro áreas, a saber: o estilo de aprendizagem, a cultura, o gênero e as inteligências preferenciais (SOUZA, TOMLINSON, 2011; TOMLINSON, 2001, 2008).

Há diversas teorias sobre estilo de aprendizagem, mas Tomlinson $(1999,2001,2008)$ se fundamenta na proposta de Dunn e Dunn $(1978,1993)$ e no conceito de inteligências múltiplas e preferenciais de Gardner (1994). Souza e Tomlinson (2011) destacam que um dos objetivos dessa 
teoria é o de chamar a atenção dos professores para a existência de múltiplos tipos de inteligência e o valor de cada uma delas, sem hierarquizá-las, ao contrário da valorização restrita e recorrente de inteligências verbal e matemática no cotidiano escolar.

A terceira área que compõe o perfil de aprendizagem dos alunos é a cultura, que Souza e Tomlinson (2011, p. 141) definem como "[...] um conjunto de atitudes, valores, normas, tradições e objetivos que caracterizam um grupo específico". A partir da compreensão da intrínseca relação entre cultura e educação escolar, torna-se necessário que o professor conheça, respeite e valorize as diferentes culturas que possam coexistir em sua sala de aula.

Enfim, as ferramentas para a realização das avaliações podem ser as mais variadas e processadas nas formas escritas, desenhadas, orais, por meio de dinâmicas ou observações registradas. Quando o professor propõe aos alunos atividades avaliativas sobre os seus interesses e perfil de aprendizagem, não somente passa a conhecer mais os alunos, como também os próprios alunos passam a refletir sobre seus próprios interesses e perfis de aprendizagem, viabilizando o autoconhecimento. Os resultados das avaliações devem ser analisados cuidadosamente pelo professor, pois orientarão a organização do ensino, ou seja, farão com que o professor responda com diferenciação.

\section{RESPONDER COM DIFERENCIAÇÃO NO ENSINO}

Responder com diferenciação significa organizar o ensino de modo flexível para atender às necessidades e especificidades dos alunos, sem deixar de considerar o conteúdo do currículo de base comum, levando em consideração o fato de que toda diferenciação deve ser realizada a partir do nível de preparação, interesses e perfil de aprendizagem dos alunos, os quais são identificados pelo professor no processo de conhecer os alunos.

Como as turmas são compostas por alunos diversos, enquanto indivíduos e grupos, para atender a cada um e ao mesmo tempo a todos, o professor utilizará diversas estratégias de ensino, de avaliação, recursos e materiais. O processo de responder com diferenciação envolve os três elementos: conteúdo, processo e produto. Conforme explicita Tomlinson (2008, p. 117): “[...] é difícil e algo pouco natural separar os elementos curriculares em conteúdo, processo e produto [...] De qualquer modo, pensar como diferenciar o ensino será mais fácil de gerenciar se examinarmos um elemento de cada vez".

Cabe destacar que, ainda que a diferenciação leve em consideração cada estudante como indivíduo, isso não induz, necessariamente, a uma abordagem individualizada do ensino, mas sim, a uma maneira de acompanhar o indivíduo no coletivo de sua turma. Além disso, acrescenta-se o fato de que, na prática, é preciso que os fatores orientadores e os elementos de diferenciação do ensino sejam articulados.

Feyfant (2016), em revisão de literatura sobre o ED, considera que há quatro dispositivos de diferenciação e sugere, para além do conteúdo, dos processos e das produções dos alunos, a possibilidade de diferenciação na estrutura ou organização dos espaços de aprendizagem a respeito da gestão da sala de aula como forma de favorecer as aprendizagens em turmas diferenciadas. A seguir serão detalhados os quatro elementos de diferenciação.

\section{Diferenciação no conteúdo}

O conteúdo refere-se ao o que se deseja que os alunos aprendam, ou seja, o que se propõe a ensiná-los, o conhecimento, a compreensão ou as habilidades almejadas. Diferenciar os 
conteúdos, entretanto, não significa necessariamente alterar ou suprimir os conteúdos preestabelecidos pelo currículo padrão, ao contrário, significa organizá-los para que os alunos se apropriem dos conhecimentos desejados utilizando seu potencial máximo. A diferenciação dos conteúdos pode ser realizada de dois modos: a) na forma como damos acesso ao que desejamos ensinar; e b) no que desejamos ensinar.

\section{Diferenciação no processo}

O processo refere-se à compreensão ou ao modo como os alunos processam o conteúdo, os conceitos e as ideias ensinadas. Ao abordar novos conteúdos, os alunos necessitam de um tempo para interpretar, significar e fazer relações com seus conhecimentos anteriores. Em geral as atividades/exercícios aplicados após um novo conteúdo ou conceito servem para estimular e possibilitar a aprendizagem dos alunos. Atividades de qualidade possibilitam que os alunos se apropriem dos novos conhecimentos, avançando os seus níveis de compreensão sobre o conteúdo abordado. Diferenciar o processo, nada mais é do que propor atividades diferenciadas que sejam, primordialmente, de qualidade. O que as torna diferenciadas é o fato de que elas possibilitam múltiplas formas de compreensão e significação dos tópicos abordados.

Em síntese, diferenciar o processo implica em respeitar as diferenças dentro dos processos de apropriação do saber e consiste nos meios utilizados para que os alunos compreendam os conteúdos visados. Assim, as diferenças entre os alunos devem ser levadas em consideração, de modo que variadas vias de acesso devem ser ofertadas para o conjunto dos estudantes, segundo seus perfis pedagógicos. Assim, a heterogeneidade dos estudantes deve corresponder à heterogeneidade das estratégias de ensino: construtivistas (metodologia de projetos, tutoria, aprendizagem cooperativa); interativas (debates em grupos e discussão); de trabalho individual (aprendizagem baseada em problemas e estudos de caso); ou magistrais (exposições e demonstrações) (FEYFANT, 2016).

A diferenciação de processo consiste em utilizar meios diferentes a fim de permitir que os estudantes se desenvolvam ao máximo e que, a partir de recursos internos próprios e daqueles que são ensinados, permita percursos de realização de aprendizagem diferentes para um mesmo espaço de tempo. Com atividades diferenciadas de qualidade, o professor possibilita que os alunos façam uma atividade de diversos modos, com diversos graus de sofisticação e em diferentes períodos, com diversos tipos e níveis de apoio e utilizando suas capacidades e as informações essenciais.

\section{Diferenciação no produto}

O produto refere-se à forma como os estudantes demonstram o que sabem, compreendem e estão aptos a fazer após o processo de ensino. Os trabalhos dos alunos são os indicadores do que eles aprenderam ou compreenderam, além de ser o meio de mostrar como eles utilizam e representam o que foi aprendido. A diferenciação do produto consiste, portanto, em prover oportunidade de escolha de apoios e de ferramentas segundo as atividades, assim como de modular ou flexibilizar o formato ou tipo de tarefa numa mesma atividade, a fim de que os alunos atinjam os objetivos de conhecimentos e competências almejados. Entretanto, é preciso evitar que os alunos trabalhem sempre com os métodos que lhes sejam mais convenientes, pois é preciso incentivar que eles se apropriem de outras estratégias. 
Diferenciação na estrutura da aula

Ao articular os fatores e os elementos de diferenciação, é necessário a reestruturação da gestão e organização da sala aula. Conforme destaca Caron (2008), a diferenciação na estrutura da aula abre a porta para a diferenciação nos outros dispositivos (conteúdo, processo e produto) e envolve a gestão das aprendizagens, o clima organizacional, o conteúdo organizacional e a organização da sala de aula.

Diferenciar a estruturação do trabalho de professores e alunos na sala de aula passa pela organização dos tempos e espaços, da disposição de carteiras/mesas e acessibilidade aos recursos para favorecer a aprendizagem dos alunos da turma. Tomlinson $(1999,2008)$ descreve que, na prática, o ED consiste em um misto de ensino para grupo-turma, pequenos grupos e individual, oferecendo a seguinte sugestão de fluxo:

- O professor e toda a turma começam a explorar um tópico ou conceito;

- Os alunos se empenham em estudos adicionais fazendo uso de materiais variados, tendo como base seu nível de preparação e estilo de aprendizagem;

- Alunos e professores se reúnem para a partilha de informações e questões;

- Alunos executam várias tarefas concebidas para que processem ideias-chave de diferentes níveis de complexidade e em diferentes ritmos;

- Toda a turma revê as ideias-chave e desenvolve estudos através do compartilhamento;

- Reunidos em pequenos grupos, os alunos aplicam os princípios-chave na resolução de problemas, concebidos pelo professor, relacionados com o tópico em questão;

- Toda a turma é preparada nas capacidades para fazer uma apresentação;

- Os alunos escolhem as áreas de interesse através das quais irão expandir seus níveis de compreensão;

- Toda a turma escuta os relatos dos planos individuais e estabelece critérios de sucesso.

Assim, uma turma diferenciada é marcada pelo ritmo repetitivo de preparação, revisão e partilha na turma-grupo, seguindo-se as oportunidades de exploração, compreensão e expansão individual ou em pequenos grupos. O ensino grupo-turma tende a ser eficaz na realização de atividades de: avaliação diagnóstica (nível de preparação, interesses e perfil de aprendizagem), introdução de conceitos, partilha e construções coletivas para síntese (TOMLINSON, 1999, 2008).

$\mathrm{O}$ ensino em pequenos grupos no $\mathrm{ED}$ se dá, em essência, pela constituição de agrupamentos flexíveis e consiste em importante estratégia de gestão da turma, além de responder à indagação de muitos professores sobre como atender responsivamente à diversidade dos alunos em uma mesma turma, ensinando a todos e cada um.

Os agrupamentos flexíveis são formados a partir dos fatores de diferenciação, ou seja, os alunos podem ser agrupados de acordo com o nível de preparação, interesses ou perfil de aprendizagem (HEACOX, 2006; TOMLINSON, 1999, 2008). Os professores podem, ainda, adotar critérios de: a) similaridade - agrupando alunos com características similares em relação aos fatores de diferenciação; b) complementariedade - agrupando alunos com características diversas em relação aos fatores de diferenciação, mas que se complementam. O critério adotado deverá ser em conformidade com a compreensão do que será mais eficaz em função dos objetivos estabelecidos para os alunos.

A flexibilidade dos agrupamentos também se dá pela quantidade de alunos que compõem os grupos, não havendo a necessidade de que os grupos sejam compostos pela mesma quantidade 
de alunos. Há de se destacar que, em grupos flexíveis, todos os alunos executam a mesma atividade, podendo ou não ser executada e/ou registrada de modo individual.

Desta forma, os agrupamentos são alterados com frequência de forma a adequar o ensino às especificidades dos alunos, a evitar qualquer forma de estigmatização, além de estimular a colaboração, contribuindo também para a compreensão de comunidade da turma, pois há interação alternada entre todos os alunos. O professor tanto pode formar grupos como, também, permitir que os próprios alunos de agrupem. A organização da sala, adotando-se ou não a estratégia de agrupamentos flexíveis, também pode ser feita na forma de ateliês ou estações de ensino, o que envolve a estruturação da sala em diferentes espaços e permite dividir as aprendizagens e tarefas em tempos mais ou menos curtos. Depois desses ateliês, pode-se demandar o reagrupamento dos alunos para uma tarefa comum à turma. O ensino se servirá desses ateliês para arranjar os apoios, auxílios ou regulações durante tarefa.

Outras sugestões práticas de diferenciação nas estruturas seriam: atingir o pré-requisito de conhecer os alunos; não se prolongar apenas no ensino dos mais frágeis; conhecer os possíveis dispositivos de diferenciação e escolher com base no que se pretende ensinar e na necessidade do aluno; modular o programa de formação, diferenciando o conteúdo; fornecer material que reflita a diversidade de culturas e modelos de famílias; trabalhar em equipe e por ciclos sob longo tempo; arranjar espaços de calma ou propícios para a colaboração; definir com os alunos o uso de modalidades diferentes de trabalho e, se necessário, permitir que eles se movam ou que busquem se acalmar, se a situação ou o aluno assim demandar.

\section{ENSINO DIFERENCIADO E ALUNOS COM NECESSIDADES EDUCACIONAIS ESPECIAIS}

Em um esforço para atingir o máximo de estudantes de uma só vez, os professores costumam dedicar a maior parte de seu planejamento e ensino à média dos alunos da turma. Entretanto, quando os alunos do público-alvo da educação especial entram na sala de aula comum, a ineficácia desse ensino homogêneo e mediano se destaca, assim como se torna mais aparente a diversidade e a heterogeneidade presente em sala de aula, além de serem vistas como um problema.

Assim, se os alunos do público-alvo da educação especial devem alcançar os níveis máximos possíveis dos padrões curriculares gerais, eles precisam aprender nas salas de aula e ter acesso ao conteúdo do currículo geral para que possam colher os benefícios das altas expectativas que são impostas para a turma como um todo. Para Lawrence-Brown (2004) a diferenciação deve ser pensada como servindo a dois objetivos gerais. O primeiro seria o de maximizar a obtenção dos padrões gerais do currículo no nível da série ou ciclo para todos os alunos e a estratégia importante para isso seria fornecer suporte adicional para alunos com dificuldades. O segundo objetivo seria o de fornecer currículos com conteúdo ajustado para os alunos que deles precisam, que poderiam ser um currículo enriquecido ou um currículo priorizado.

Alunos superdotados e talentosos podem se beneficiar de oportunidades mais desafiadoras e criativas que vão além dos padrões do currículo comum. No caso dos alunos com deficiências, o Plano Educacional Individualizado (PEI) pode equilibrar entre os objetivos acadêmicos do currículo comum com habilidades mais funcionais e, quando o conteúdo deve ser limitado em função do tempo necessário para promover as aprendizagens, o PEI (TANNÚS-VALADÃO, MENDES, 2018) deve indicar o que priorizar na diferenciação do conteúdo.

Há na literatura várias estratégias de ensino que podem ser implementadas na sala de aula para promover a diferenciação e o ensino diferenciado é uma dessas propostas, assim como o modelo

Revista Teias v. 22 • n. 66 • jul./set. 2021 • Seção temática Programas e práticas pedagógicas na educação especial e inclusiva 
do Desenho Universal para a Aprendizagem (DUA). A distinção entre a AED e o DUA reside no fato de que a AED propõe que as estratégias de ensino atendam as necessidades individuais do estudante, identificadas através de uma avaliação formativa, enquanto o DUA propõe que o ensino seja propositalmente delineado para atender a todos os estudantes (WASA SCHOOL INFORMATION AND RESEARCH SERVICE, 2018). Assim, a AED busca balancear as necessidades comuns a todos os estudantes com necessidades individuais mais específicas dos aprendizes, demonstrando que as duas abordagens não são incompatíveis e podem ser associadas.

Como em qualquer outro esforço de mudança de escola, Lawrence-Brown (2004) recomenda, ainda, que se evite resultados inesperados no uso da AED e ressalta que este pode ser um processo mais fácil se o professor não entrar em pânico diante de todas as possibilidades de diferenciações possíveis, uma vez que nem todos esses suportes nas diferenciações serão necessários para todas as situações. Além disso, não se deve tentar fazer tudo de uma vez, pois se o professor se deixar sobrecarregar com tudo o que gostaria e poderia diferenciar, ele provavelmente nunca fará nada e ficará insatisfeito, uma vez que as possibilidades de diferenciação podem ser ilimitadas e, assim, priorizar sempre será uma necessidade.

Adicionalmente, a busca de colaboração pode tornar o progresso mais rápido e parcerias podem e devem ser estabelecidas para sustentar a AED, podendo ser estabelecidas com bibliotecários, coordenadores, auxiliares, professores (de séries acima e abaixo, da área de educação especial e especialistas em matérias do currículo) etc. A criação de um acervo, real ou virtual, de materiais e recursos para diversificar o ensino pode facilitar o trabalho do professor.

\section{CONSIDERAÇÕES FINAIS}

A AED, como outros modelos que visam favorecer a inclusão escolar, é uma proposta de apoio à sala de aula como uma comunidade, portanto universal, que beneficia todos os estudantes com ou sem deficiências, talentos especiais e superdotação. Considerando-se que a literatura sobre inclusão escolar recomenda que novas formas de se pensar o ensino em classes heterogêneas sejam testadas, a fim de que as diferenças sejam respeitadas, a proposta da AED vem sendo reforçada como uma dessas abordagens indicadas, sendo este um referencial importante para a formação de professores para a inclusão escolar. Sugere-se ainda que a produção cientifica nacional sobre inclusão escolar explore as perspectivas de diálogo com os estudos curriculares e as diferenças humanas presentes em nossas salas de aula.

\section{REFERÊNCIAS}

CARON, Jacqueline. Différencier au quotidien: Cadre d'expérimentation avec points de repères et outils-support. Montréal: Chenelière Éducation, 2008.

DUNN, RITA; DUNN, Kenneth. Teaching students through their individual learning styles: a practical approach. Reston, VA: Reston Publishing Co., 1978

DUNN, Rita; DUNN, Kenneth. Teaching secondary students through their individual learning styles: practical approaches for grades. p. 7-12. Boston: Allyn \& Bacon, 1993.

FEYFANT, Annie. La différenciation pédagogique en classe. Dossier de veille de l'IFÉ, n. 113. Novembre 2016. Disponível em http://veille-et-analyses.ens-lyon.fr/DA-Veille/113- novembre2016.pdf. Acesso em 16 set. 2019. 
GARDNER, Howard. Estruturas da mente - a teoria das inteligências múltiplas, Porto Alegre: Artes Médicas Sul, 1994.

HEACOX, Diane. Diferenciação curricular na sala de aula. Portugal: Porto Editora, 2006.

LAWRENCE-BROWN, Diana. Differentiated Instruction: Inclusive Strategies for StandardsBased Learning That Benefit The Whole Class. American Secondary Education, v. 32, n. 3, p. 34-62. 2004. Disponível em www.jstor.org/stable/41064522. Acesso em 23 maio 2020.

PERRENOUD, Philippe. Pedagogia diferenciada. Das intenções à ação. Porto Alegre: Artmed Ed., 1999.

PRESTES, Zoia R. Quando não é a mesma coisa: análise de traduções de Lev Semionovitch Vigotski no Brasil: repercussões no campo educacional. 295 f. Tese (Doutorado em Educação) Universidade de Brasília. Brasília, Distrito Federal, 2010.

ROLDÃO, Maria do Céu. Diferenciação curricular revisitada. Conceito, discurso e práxis. Porto: Porto Editora, 2003.

SOUSA, David A.; TOMLINSON, Carol Ann. Differentiation and the Brain: How Neuroscience Supports the Learner-Friendly Classroom (Use Brain-Based Learning and Neuroeducation to Differentiate Instruction). Alexandria, VA: ASCD, 2010.

SOUZA, David; TOMLINSON, Carol Ann. Differentiation and the brain: how neurosciense supports the learner-friendly. Bloomington: Solution Tree Press, 2011.

TANNÚS-VALADÃO, Gabriela; MENDES, Enicéia G. Inclusão escolar e o planejamento educacional individualizado: estudo comparativo sobre práticas de planejamento em diferentes países. Rev. Bras. Educ., Rio de Janeiro, v. 23, e230076, 2018. Disponível em https://www.scielo.br/scielo.php? script $=$ sci abstract\&pid $=S 1413-$

24782018000100261\&lng=en\&nrm=iso\&tlng=pt. Acesso em 16 abr. 2021.

TOMLINSON, Carol Ann. The differentiated classroom: Responding to the needs of all learners. Alexandria, VA: ASCD, 1999.

TOMLINSON, Carol Ann. How to differentiate instruction in mixed-ability classrooms. 2. ed. Alexandria, VA: ASCD, 2001.

TOMLINSON, Carol Ann. Fulfilling the promise of the differentiated classroom: Strategies and tools for responsive teaching. Alexandria, VA: ASCD, 2004.

TOMLINSON, Carol Ann. The Differentiated Classroom: Responding to the Needs of All Learners 2. ed. Alexandria, VA: ASCD, 2016.

TOMLINSON, Carol Ann. How to Differentiate Instruction in Academically Diverse Classrooms. 3. ed. Alexandria, VA: ASCD, 2017.

TOMLINSON, Carol Ann; BRIMIJOIN, Kay; NARVAEZ, Lane. The differentiated school: making revolutionary changes in teaching and learning. Alexandria, VA: ASCD, 2008.

TOMLINSON, Carol Ann; BRIGHTON, Catherine; HERTBERG, Holly; CALLANHAN, Carolyn M.; MOON, Tonya R.; BRIMIJOIN, Kay; CONOVER, Lynda A.; REYNOLDS, Timothy. Differentiating Instruction in Response to Students Rediness, Interest, and Learning Profile in Academically Diverse Classrooms: a Review of Literature. Journal for the Education of the Gifet. v. 27, n. 2/3, p. 119-145, 2003.

TOMLINSON, Carol Ann; MCTIGHE, Jay. Integrating differentiated instruction \& understanding by design: Connecting content and kids. Alexandria, VA: ASCD, 2006. 
WASA SCHOOL INFORMATION AND RESEARCH SERVICE. Best practices for Differentiated Instruction, Hanover Research, 2018. Disponível em http://www.wasaoly.org/WASA/images/WASA/1.0\%20Who\%20We\%20Are/1.4.1.6\%20SIRS/Download_Files /LI\%202018/Mar-Best\%20Practics\%20for\%20Differentiated\%20Instruction.pdf. Acesso em 18 abr. 2021.

\section{Informações das autoras}

Keisyani da Silva Santos

Universidade Federal de São Carlos

E-mail:keisyani@gmail.com

ORCID: http://orcid.org/0000-0002-1569-5234

Link Lattes: http://lattes.cnpq.br/9429520073186592

Enicéia Gonçalves Mendes

Universidade Federal de São Carlos

E-mail: eniceia.mendes@gmail.com

ORCID: http://orcid.org/0000-0003-3673-0681

Link Lattes: http://lattes.cnpq.br/3897627554738983 